\title{
Height and Roughness Measurement of Biomaterial's Surfaces using Scanning Electron Microscopy
}

\section{Douderova ${ }^{1}$, L. Bacakova ${ }^{2}$, V. Stary ${ }^{1,}$ P. Buescher ${ }^{3}$ and M. Bode ${ }^{4}$}

1: Department of Materials Engineering, Faculty of Mechanical Engineering, Czech Technical University in Prague, Karlovo Namesti 13, 12135 Praha 2, Czech Republic 2: Institute of Physiology, Academy of Sciences of the Czech Republic, Videnska 108314220 Praha 4, Czech Republic

3: Soft Imaging System GmbH, Johann-Krane-Weg 39, 48149 Muenster, Germany 4: Soft Imaging System Corp., Lakewood, CO 80228, USA

Biomaterials are materials of natural or man-made origin that are used to direct, supplement, or replace the functions of living tissues of the human body. Medical practice today utilizes a large number of biomaterials in the form of implants (sutures, bone plates, joint replacements, ligaments, vascular grafts, heart valves, intra-ocular lenses, dental implants, etc.) and medical devices (pacemakers, biosensors, artificial hearts, blood tubes, etc.) which are widely used to replace and/or restore the function of traumatized or degenerated tissues or organs, to assist in healing, to improve function, to correct abnormalities, and thus improve the quality of life of the patients.

The contact of the biomaterial with the live tissue is influenced by the chemical, morphological and other properties of materials and their surfaces. The most important morphological properties are the topography, the porosity and the roughness of the surface.

The aim of this study was to use the Scandium software for comparison of two different surface modifications: electro erosion and plasma spray technique and to find out the common dependence of cell growth on the roughness of the implant surfaces. The priority of this work is measuring of the surface roughness and its evaluation. We were successful in finding the dependence of number of cells adhered after one day on the roughness parameter $\mathrm{Ra}$ and the dependence of the doubling time on Rsk parameter.

The images were acquired using the Scanning Electron Microscope JSM-5410 and analyzed with the software Scandium Solution Height. This new kind of surface testing runs without any contact with the material. That is a great advantage for very soft materials like the plastics or pure soft metals as well as very thin and soft surface layers which can be scratched during conventional measurements with the contact profilometer. Another advantage is that Scandium and its Solution Height can be integrated into an existing laboratory computer environment and does not need new measuring hardware equipment. 